\title{
Measurements of Galactic Cosmic-Ray Hydrogen and Helium Isotopes with the BESS-Polar II Instrument
}

\author{
Nicolas Picot-Clémente*, K. Abe ${ }^{2}$, H. Fuke ${ }^{3}$, S. Haino ${ }^{4}$, T. Hams ${ }^{5}$, M. Hasegawa ${ }^{4}$, A. \\ Horikoshi $^{4}$, A. Itazaki ${ }^{2}$, K.C. Kim ${ }^{1}$, T. Kumazawa ${ }^{4}$, A. Kusumoto ${ }^{2}$, M.H. Lee ${ }^{1}$, Y. \\ Makida $^{4}$, S. Matsuda ${ }^{4}$, Y. Matsukawa ${ }^{2}$, K. Matsumoto ${ }^{4}$, J.W. Mitchell ${ }^{5}$, A.A. Moiseev ${ }^{5}$, \\ J. Nishimura ${ }^{1}$, M. Nozaki ${ }^{4}$, R. Orito ${ }^{2}$, J.F. Ormes ${ }^{6}$, K. Sakai ${ }^{5}$, M. Sasaki ${ }^{5}$, E.S. Seo ${ }^{1}$, Y. \\ Shikaze $^{2}$, R. Shinoda ${ }^{7}$, R.E. Streitmatter ${ }^{5}$, J. Suzuki ${ }^{4}$, Y. Takasugi ${ }^{2}$, K. Takeuchi' ${ }^{2}$, K. \\ Tanaka $^{4}$, N. Thakur ${ }^{6}$, T. Yamagami ${ }^{4}$, A. Yamamoto ${ }^{4}$, T. Yoshida ${ }^{3}$, K. Yoshimura ${ }^{4}$ \\ 1 Institute for Physical Science and Technology, University of Maryland, College Park MD 20742, \\ USA \\ 2 Kobe University, Kobe, Hyogo 657-8501, Japan \\ 3 Institute of Space and Astronautical Science, Japan Aerospace Exploration Agency \\ (ISAS/JAXA), Sagamihara, Kanagawa 252-5210, Japan \\ 4 High Energy Accelerator Research Organization (KEK), Tsukuba, Ibaraki 305-0801, Japan \\ 5 National Aeronautics and Space Administration, Goddard Space Flight Center (NASA/GFSC), \\ Greenbelt, MD 20771, USA \\ 6 University of Denver, Denver, CO 80208, USA \\ 7 The University of Tokyo, Bunkyo, Tokyo 113-0033, Japan
}

\begin{abstract}
The Balloon-Borne Experiment with a Superconducting Spectrometer (BESS-Polar II) flew successfully over Antarctica for 24.5 days in December 2007 through January 2008 during a period of minimum solar activity. BESS-Polar II is concentrically arranged with various particle detectors and a solenoidal superconducting magnet that allow it to accurately identify hydrogen and helium isotopes among the incoming Galactic cosmic-ray nuclei. ${ }^{2} \mathrm{H}$ and ${ }^{3} \mathrm{He}$ are mostly secondary particles produced after interaction of primary ${ }^{1} \mathrm{H}$ and ${ }^{4} \mathrm{He}$ cosmic rays during their propagation in the interstellar medium. The high mass separation of BESS-Polar II, the long-duration balloon flight and the stability of the instrument provide unprecedented high precision measurements of light isotopes. This paper will present new measurements of hydrogen and helium isotopes with energies from $0.2 \mathrm{GeV} /$ nucleon up to about $1.5 \mathrm{GeV} /$ nucleon, which gives essential information to better understand the history of cosmic-ray propagation in the Galaxy. They will be compared to previous results and theoretical calculations using GALPROP.
\end{abstract}

The 34th International Cosmic Ray Conference,

30 July- 6 August, 2015

The Hague, The Netherlands

\footnotetext{
*Speaker.

${ }^{\dagger}$ Email: picot@umd.edu
} 


\section{Introduction}

This paper reports precise measurements of cosmic-ray hydrogen and helium isotopes with the BESS-Polar II instrument which flew in Antarctica for 24.5 days in December 2007 through January 2008. BESS-Polar II is the second long-duration balloon flight in Antarctica following BESS-Polar I in 2004. BESS-Polar flights are the successor of the 9 previous BESS campaigns that successfully took place in the Northern hemisphere from 1993 to 2002 [U].

Secondary particles of ${ }^{2} \mathrm{H}$ and ${ }^{3} \mathrm{He}$ mostly come from the interaction of primary hydrogen (through fusion reaction for ${ }^{2} \mathrm{H}$ ) and helium nuclei (through fragmentation for ${ }^{2} \mathrm{H}$ and ${ }^{3} \mathrm{He}$ ) in the interstellar medium. As shown in [[]], secondary-to-primary ratio and flux measurements of hydrogen and helium isotopes can be as constraining as the widely used Boron-to-Carbon ratio on cosmic-ray propagation models and parameters. The study of light isotope propagation can also probe the universality of propagation of light and heavy nuclei. The BESS spectrometer allows to separate and measure cosmic-ray hydrogen $\left({ }^{1} \mathrm{H}\right.$ and $\left.{ }^{2} \mathrm{H}\right)$ and helium $\left({ }^{3} \mathrm{He}\right.$ and $\left.{ }^{4} \mathrm{He}\right)$ isotopes from 0.2 to $1.5 \mathrm{GeV} /$ nucleon [3], 田, 回], which gives crucial information to understand the propagation history of cosmic rays in the Galaxy.

BESS-Polar is a multipurpose particle instrument. Besides measuring light isotopes, the spectrometer is also capable of precisely measuring low-energy antiprotons [5], searching for antinuclei

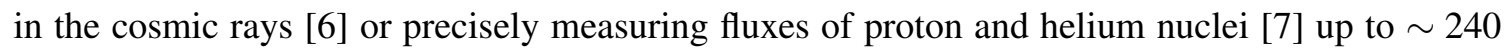
$\mathrm{GV}$, which is the Maximum Detectable Rigidity (MDR) ${ }^{1}$. BESS-Polar II flew during a solar minimum over Antarctica at low rigidity cut-off. 4.7 billion particles were recorded during its stable and long-duration balloon flight, more than all previous BESS cumulated flights. The instrument and flight will be presented in Section $\S \square$ and the analysis will be described in Section $\S$ [. The precise measurements of hydrogen and helium isotopes will finally be given and discussed in section $\S$ 田.

\section{The BESS-Polar Instrument}

The BESS-Polar II spectrometer is presented in Figure $\mathrm{W}$. The instrument is concentrically arranged with various particle detectors and a solenoidal superconducting magnet. A particle crossing the instrument from top to bottom will pass through a top time-of-flight hodoscope (TOF), made of plastic scintillators with photomultipliers (PMT) at both ends, a magnet operated at 0.8 Tesla, a drift-type tracking system, a middle TOF counter, an aerogel Cherenkov counter used to separate antiprotons from leptons ${ }^{2}$, and a bottom TOF counter.

The difference in the measured times when a particle passes the top and bottom (or top and middle) TOFs gives the velocity $\beta$ of the crossing particle. A timing resolution of $120 \mathrm{ps}$ is achieved for singly-charged particles, yielding a resolution on $\beta^{-1}$ of $2.5 \%$. Ionization energy losses $d E / d x$ in the TOF paddles give the particle electric charge $Z$. Using hits in the drift-type tracking system, made of 4 layers of inner drift chambers (IDC) and a central JET-type drift chamber, the track of the particle deviated by the magnetic field is reconstructed and its rigidity $R$ can be derived. A magnetic rigidity resolution of about $0.4 \%$ at $1 \mathrm{GV}$ and a MDR of $240 \mathrm{GV}$ are achieved with the tracking system. More detailed information about the BESS-Polar detector can be found in [Q, ए0, प].

\footnotetext{
${ }^{1}$ Beyond the Maximum Detectable Rigidity, curved particle paths can't be distinguished from straight lines.

${ }^{2}$ It rejects $e^{-}$and $\mu^{-}$with a factor of 12000 to identify antiprotons up to $3.5 \mathrm{GeV}$.
} 


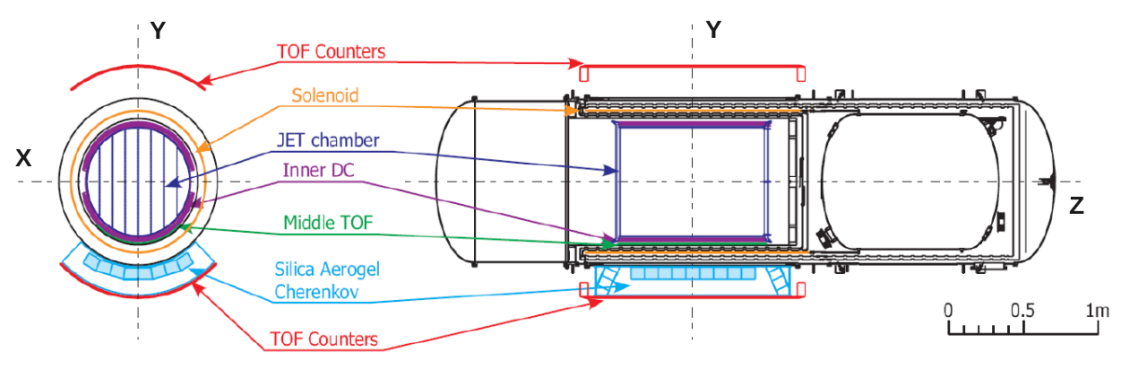

Figure 1: Cross-sectional views of the BESS-Polar II payload.

From these measurements, the mass $M$ of the crossing particle can be obtained with:

$$
M=Z R \sqrt{1 / \beta^{2}-1}
$$

The particle rigidity is measured inside the magnetic spectrometer, and its corresponding kinetic energy $E_{i}$ is:

$$
E_{i}=\sqrt{R^{2} Z^{2}+M^{2}}-M
$$

Its kinetic energy at top of instrument is determined by correcting the measured energy for losses in the different layers in the upper part of the instrument. The energy at top of atmosphere is obtained by correcting the energy at top of instrument for ionization energy losses in the atmosphere.

Flying over Antarctica ensures that measurements were made at low rigidity cut-offs, with a majority of the BESS-Polar II flight below $0.2 \mathrm{GV}$ and a maximum at $0.6 \mathrm{GV}$. The instrument flew between 22 December 2007 and 21 January 2008 at an altitude between $34 \mathrm{~km}$ and $38 \mathrm{~km}$ (residual average atmosphere of $5.8 \mathrm{~g} \mathrm{~cm}^{-2}$ ). 2 out of 44 TOF PMTs were turned off during the flight due to high voltage issues, and calibration of the tracker over short periods of time had to be performed due to high voltage fluctuations that occurred in the central tracker.

\section{Data Analysis}

For the isotope analysis, GEANT3 Monte Carlo [[2] simulations of particles crossing the instrument were performed. 800 million downward-going hydrogen ${ }^{1} \mathrm{H},{ }^{2} \mathrm{H}$ and helium ${ }^{3} \mathrm{He},{ }^{4} \mathrm{He}$ particles were simulated isotropically with energies ranging from $0.001 \mathrm{GeV}$ to $25 \mathrm{GeV}$. The simulations are used to determine the geometric acceptance of the instrument and interaction efficiencies.

In order to separate hydrogen and helium isotopes, a rigorous event selection is required to achieve precise measurements of $\beta, Z$ and $R$, and remove hadronic interacting events. In this analysis, measurements of $\beta$ are made using only the combination of top and bottom TOFs to get the best precision. This defines the energy threshold of the isotope analysis of $0.2 \mathrm{GeV} /$ nucleon, below which particles can be stopped in the instrument due to ionization energy losses. For best TOF $d E / d x$ measurements, the two TOF paddles (out of 22) containing only one working PMT are excluded. These preliminary cuts define the geometric acceptance of the detector and is about $0.29 \mathrm{~m}^{2} \mathrm{sr}$ at $1 \mathrm{GeV} /$ nucleon. 
Among the particles passing preliminary cuts, we first select those with $Z=1$ or $Z=2$ at the top TOF using $d E / d x$ measurements. Multiple selection criteria are then needed to separate isotopes. They are divided into two categories:

- The "single-track" cuts are aimed to remove hadronic interacting events and ensure that particles are passing through the fiducial region of the JET chamber. They reject events with multiple reconstructed tracks inside the instrument and more than 2 hits in the top and bottom TOFs. In addition, only particle's track for which at least 32 hits are expected in the JET chamber are kept. Finally, to remove remaining hadronic interacting events and cross check TOF $d E / d x$ measurements, the bottom TOF is also used to select $Z=1$ or $Z=2$ particles.

- The "quality" cuts are used to remove badly reconstructed events due to noise or detector limitations. They ask reconstructed tracks with $\chi^{2}<5$ in both XY and YZ detector planes and an estimated error on the inverse rigidity $\Delta R^{-1}<0.015$. Only particles with less than 100 hits in the JET chamber and with a minimum track length of $500 \mathrm{~mm}$ in the XY plane are kept. In addition, other criteria include at least 3 out of 4 vernier pads (in each layer of IDC), and good consistency between reconstructed track and hit position in the TOFs $(<75$ $\mathrm{mm})$.

After refining flight data with the single-track and quality selection cuts, we can now separate hydrogen and helium isotopes. Figure $\square$ illustrates the high mass separation of the BESS-Polar II instrument, and shows the good agreement between theoretical lines and measurements which indicates that both $\beta$ and $R$ are well measured. At the lowest rigidities, discrepancies observed between measurements and theoretical lines reflect detection limitations of BESS-Polar II.

Particle's mass distributions obtained from measurements of rigidity $R$, velocity $\beta$ and charge $Z$ are used to identify and count each isotope species. They are more difficult to distinguish with increasing energy and we use double Gaussian distributions to fit mass histograms at the highest energies. Hydrogen and helium isotopes can be separated and counted up to $\sim 1.5 \mathrm{GeV} /$ nucleon.

\section{Results}

Secondary-to-primary ratios such as ${ }^{2} \mathrm{H} /{ }^{1} \mathrm{H}$ and ${ }^{3} \mathrm{He} /{ }^{4} \mathrm{He}$ give important information on propagation history of cosmic rays in the interstellar medium and are independent on the unknown source spectra. Measurements of ${ }^{2} \mathrm{H} /{ }^{1} \mathrm{H}$ and ${ }^{3} \mathrm{He} /{ }^{4} \mathrm{He}$ provide the advantage to be quasi insensitive to the acceptance, efficiencies and atmospheric corrections, and thus to their uncertainties, as they are similar for same charged particles. In Figure 1$]$ and [A, BESS-Polar II measurements of these secondary-to-primary ratios between $0.2 \mathrm{GeV}$ /nucleon and $1.5 \mathrm{GeV} /$ nucleon are presented and compared to previous measurements from IMAX-92 [ㅍ]], BESS-93 [3]], AMS-01 [प15], BESS00 [四] and PAMELA [ए6]. A modified version of GALPROP [17], in which the proton fusion reaction was implemented and production cross sections were modified (c.f. [1[8]), was computed to compare results with the "Plain Diffusion" (dashed lines) and "Reacceleration" (solid lines) models, with default diffusion and injection parameters from WebRun ${ }^{3}$ [एप] , using different solar modulations of 400, 800 and $1200 \mathrm{MV}$.

\footnotetext{
${ }^{3}$ GALPROP WebRun is available at http://galprop.stanford.edu/
} 

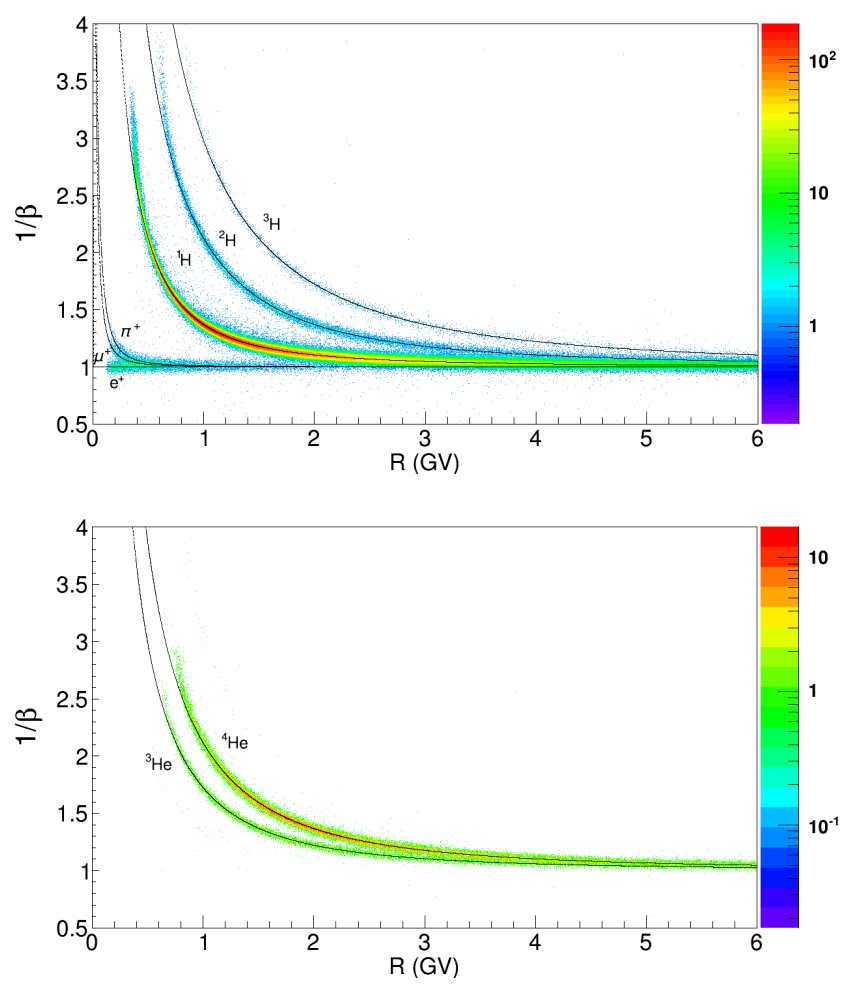

Figure 2: $\beta^{-1}$ versus $R$ for $Z=1$ (top panel) and $Z=2$ (bottom panel) particles after applying the event selection cuts, for $45 \mathrm{~min}$ of data taking. Dashed lines correspond to the theoretical curves for various species present in the data.

Measurements from BESS-Polar II and PAMELA were taken during the same solar minimum, and similar results for ratios are expected for both experiments. BESS-Polar $\mathrm{II}^{2} \mathrm{H} /{ }^{1} \mathrm{H}$ is consistent with PAMELA within uncertainties. ${ }^{2} \mathrm{H} /{ }^{1} \mathrm{H}$ results follow solar modulation expectations which predict low ratios at solar minimum and high ratios at solar maximum (observed with IMAX92 and BESS-00). Although a slighly higher ratio was expected for the BESS-93 ratio ${ }^{2} \mathrm{H} /{ }^{1} \mathrm{H}$ (taken near solar minimum), measurements are similar to BESS-Polar II and PAMELA, however they are subject to large uncertainties. Secondary-to-primary ratios of ${ }^{3} \mathrm{He} /{ }^{4} \mathrm{He}$ are less affected by solar modulations due to similar A/Z between the two species. BESS-Polar II ${ }^{3} \mathrm{He} /{ }^{4} \mathrm{He}$ is, as expected, consistent within uncertainties with previous measurements from BESS-93 and AMS-01 taken close to a solar minimum. Discrepancies are however observed with PAMELA data, that are significantly lower and close to IMAX-92, taken near solar maximum.

The GALPROP program [ㄱ] is a numerical code aimed to calculate cosmic-ray propagation in the Galaxy by incorporating as many processes and astrophysical data as possible. For comparison, we used the modified version of GALPROP from [18], more suitable for studying hydrogen and helium isotopes, in which authors implemented the proton fusion interaction and updated fragmentation cross sections. Injection spectra are power laws in momentum $d q(p) / d p \propto p^{-\gamma}$ in GALPROP, and the spatial diffusion coefficient is defined as $D_{x x}=\beta D 0_{x x}\left(\rho / \rho_{0}\right)^{\delta}$, where $\beta$ is the speed-of-light divided velocity, $\rho$ is the magnetic rigidity, and $D 0_{x x}, \delta$ are diffusion parameters. 


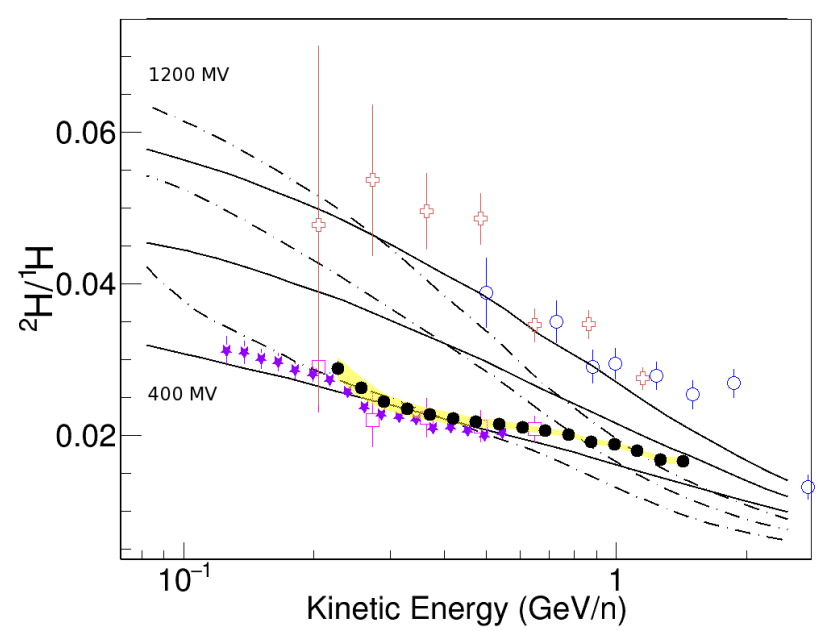

Figure 3: Our measured ${ }^{2} \mathrm{H} /{ }^{1} \mathrm{H}$ ratio at top of atmosphere with BESS-Polar II (black filled circles). The error bars show statistical uncertainties while the yellow shaded region shows the systematic uncertainties. Measurements are compared with IMAX-92 [ㄴ]] (blue open circles), BESS-93 [B]] (pink open squares), BESS00 [团] (red open crosses) and PAMELA [ए]6] (purple filled stars). A modified version of GALPROP [ए]7], in which the proton fusion reaction was implemented and production cross sections were modified (c.f. [[8]), is employed for comparison using the "Plain Diffusion" (dashed lines) and "Reacceleration" (solid lines) models, with default diffusion and injection parameters from WebRun [ए耳], at different solar modulation parameters of 400, 800 and $1200 \mathrm{MV}$.

The code was computed using two different models from WebRun [ㅁ]. The "Plain Diffusion" model uses the diffusion parameters $D 0_{x x}=2.2 \times 10^{28} \mathrm{~cm}^{2} \mathrm{~s}^{-1}, \delta_{1}=0$ and $\delta_{2}=0.6$ above and below $\rho_{0}=3 \mathrm{GV}$, and the injection parameters $\gamma_{1}=2.30$ below $40 \mathrm{GV}$ and $\gamma_{2}=2.15$ above. The "Reacceleration" model uses an Alfvén speed of $36 \mathrm{~km} \mathrm{~s}^{-1}$ for the reacceleration process, $D 0_{x x}=5.75 \times 10^{28} \mathrm{~cm}^{2} \mathrm{~s}^{-1}, \delta=0.34$ and $\rho_{0}=4 \mathrm{GV}$ as diffusion parameters, and $\gamma_{1}=1.82$ below and $\gamma_{2}=2.36$ above $9 \mathrm{GV}$ as injection parameters.

Measurements from IMAX-92, BESS-93 and AMS-01 are subject to large uncertainties and it was previously difficult to discriminate among different models. The new precise results of ${ }^{2} \mathrm{H} /{ }^{1} \mathrm{H}$ and ${ }^{3} \mathrm{He} /{ }^{4} \mathrm{He}$ from BESS-Polar II can now provide strong constraints on cosmic-ray propagation models and parameters. The Reacceleration model used in this paper, from a modified version of GALPROP [ए8], agrees well with both secondary-to-primary ratios with solar modulation parameter of $\sim 400 \mathrm{MV}$, while the Plain Diffusion model decreases with increasing energy faster than ${ }^{2} \mathrm{H} /{ }^{1} \mathrm{H}$ data and is significantly higher than ${ }^{3} \mathrm{He} /{ }^{4} \mathrm{He}$ BESS-Polar II measurements. There is however room to improve both models for which we have not attempted to optimize diffusion or injection parameters in order to better fit data.

\section{Conclusion}

The BESS-Polar II experiment offers unique data to significantly improve our knowledge on cosmic-ray propagation in the Galaxy. In this paper were presented cosmic-ray hydrogen and helium isotope measurements of secondary-to-primary ratios with BESS-Polar II. Results are the 


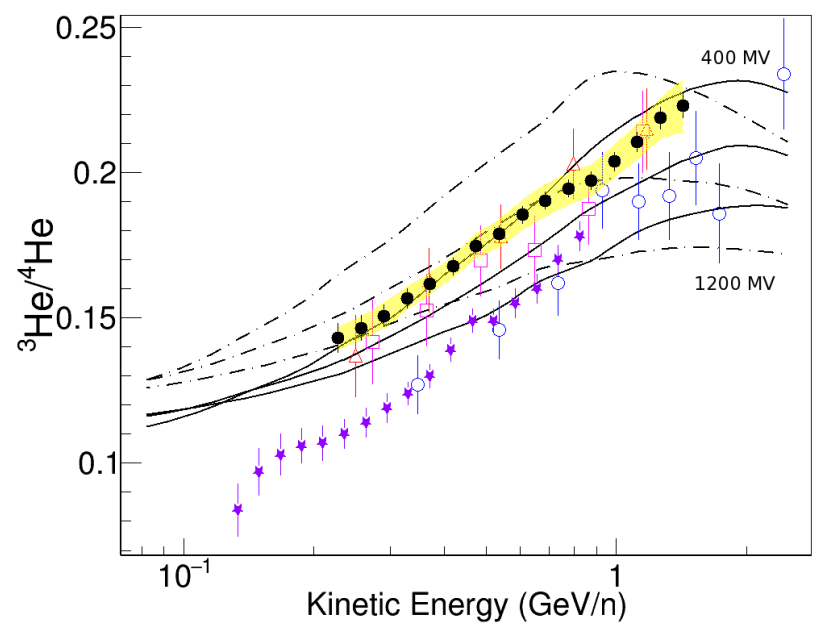

Figure 4: Our measured ${ }^{3} \mathrm{He} /{ }^{4} \mathrm{He}$ ratio at top of atmosphere with BESS-Polar II (black filled circles). The error bars show statistical uncertainties while the yellow shaded region shows the systematic uncertainties. Measurements are compared with IMAX-92 [[4]] (blue open circles), BESS-93 [B]] (pink open squares), AMS-01 [ㅍ]] (red open triangles) and PAMELA [ए]] (purple filled stars). A modified version of GALPROP [[D], in which the proton fusion reaction was implemented and production cross sections were modified (c.f. [ए8]), is employed for comparison using the "Plain Diffusion" (dashed lines) and "Reacceleration" (solid lines) models, with default diffusion and injection parameters from WebRun [ए耳], at different solar modulation parameters of 400, 800 and $1200 \mathrm{MV}$.

most precise to date between $0.2 \mathrm{GeV} /$ nucleon and $1.5 \mathrm{GeV} /$ nucleon, and show a good consistency with previous measurements and solar modulations, except with PAMELA ${ }^{3} \mathrm{He} /{ }^{4} \mathrm{He}$. For comparison, the Plain Diffusion and Reacceleration models were computed with default diffusion and injection parameters from WebRun, using a modified version of GALPROP more suitable for studying the light-quartet isotopes. As of now, without attempting to optimize these parameters, the Reacceleration model fits best both ${ }^{2} \mathrm{H} /{ }^{1} \mathrm{H}$ and ${ }^{3} \mathrm{He} /{ }^{4} \mathrm{He}$ measurements. The precision of the new BESS-Polar II results on hydrogen and helium isotopes bring now new opportunities to better constrain cosmic-ray propagation models and parameters.

\section{Acknowledgements}

This work was supported by NASA grant NNX15AC16G and its predecessors. The BESSPolar experiment is a U.S.-Japan collaboration supported by NASA in the US and KAKEN-Hi in Japan. The authors thank NASA Headquarters, the NASA Balloon Program Office at Goddard Space Flight Center/Wallops Flight Facility, and the NASA Columbia Scientific Balloon Facility, as well as ISAS/JAXA and KEK for their support. Thanks to the US National Science Foundation (NSF) and Raytheon Polar Services Company for their professional support in the USA and in Antarctica. Thanks to the GALPROP team, supported through NASA grants and by the Max Planck Society, for providing the program to the scientific community (http://galprop.stanford.edu/). 


\section{References}

[1] A. Yamamoto et al., Search for cosmic-ray antiproton origins and for cosmological antimatter with BESS, Adv. Space Res. 51 (2), 227, 2013.

[2] B. Coste, L. Derome, D. Maurin and A. Putze, Constraining Galactic cosmic-ray parameters with $Z$ $\leq 2$ nuclei, A\&A 539, A88, 2012.

[3] J. Z. Wang et al., Measurement of cosmicray hydrogen, helium, and their isotopic composition with the BESS experiment, Astrophys. J. 564, 244, 2002.

[4] K.C. Kim et al., Cosmic ray 2H/1H ratio measured from BESS in 2000 during solar maximum, Adv. Space Res. 51 (2), 234, 2013.

[5] K. Abe et al., Time variations of cosmic-ray helium isotopes with BESS-Polar I, Adv. Space Res., Adv. Space Res. 53 (10), 1426, 2014.

[6] K. Abe et al., Measurement of the cosmic-ray antiproton spectrum at solar minimum with a long-duration balloon flight over Antarctica, Phys. Rev. Lett. 108, 051102, 2012.

[7] K. Abe et al., Measurements of cosmic-ray proton and helium spectra from the BESS-Polar long-duration balloon flights over Antarctica, astro-ph, arXiv:1506.01267.

[8] K. Abe et al., Search for antihelium with the BESSpolar Spectrometer, Phys. Rev. Lett. 108, 131301, 2012.

[9] S. Haino et al., Progress of the BESS Superconducting Spectrometer, Nucl. Instrum. Methods A518, $167,2004$.

[10] T. Yoshida et al., BESS-polar experiment, Adv. Space Res. 33 (10), 75, 2004.

[11] K. Abe, H. Fuke, S. Haino, et al. Measurement of the cosmic-ray low-energy antiproton spectrum with the first BESS-polar Antarctic flight, Phys. Lett. B670, 103, 2008.

[12] Application Software Group, GEANT, Detector description and simulation tool, CERN program library long writeup W5013, 1993. http://wwwasd.web.cern.ch/wwwasd/geant/

[13] P. Papini, C. Grimani, S. A. Stephens, An estimate of the secondary-proton spectrum at small atmospheric depths, Nuovo Cimento, C19, 3, 1996.

[14] W. Menn et al., The Absolute Flux of Protons and Helium at the Top of the Atmosphere Using IMAX, Astrophys. J. 533, 281, 2000.

G.A. De Nolfo et al., A measurement of cosmic ray deuterium from 0.5-2.9 GeV/nucleon, AIP Proc. 528, 425, 2000.

[15] M. Aguilar et al., Isotopic Composition of Light Nuclei in Cosmic Rays: Results From AMS-01, Astrophys. J. 736, 105, 2011.

[16] O. Adriani et al., Measurement of the Isotopic Composition of Hydrogen and Helium Nuclei in Cosmic Rays with the PAMELA Experiment, Astrophys. J. 770, 2, 2013.

[17] A. W. Strong, I. V. Moskalenko, Propagation of Cosmic-Ray Nucleons in the Galaxy, Astrophys. J. 509, 212, 1998.

[18] N. Picot-Clémente, E. S. Seo, A. Strong, Y. Lu, Study of Cosmic-Ray Transport with the GALPROP Code, Proc. 34rd Int. Cosmic Ray Conf., ID 1267, 2015.

[19] A. E. Vladimirov et al., GALPROP WebRun: an internet-based service for calculating galactic cosmic ray propagation and associated photon emissions, astro-ph, arXiv:1008.3642. 